\title{
Differences between migrants and Spanish-born population through the HIV care cascade, Catalonia: an analysis using multiple data sources
}

\author{
J. REYES-URUEÑA ${ }^{1,2,3 *}$, C. CAMPBELL ${ }^{1,2}$, C. HERNANDO ${ }^{1,2,3}$, N. VIVES ${ }^{1,2,3,4}$,

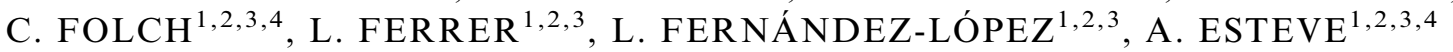 \\ AND J. CASABONA ${ }^{1,2,3,4}$ \\ ${ }^{1}$ Centre for Epidemiological Studies on HIVISTI of Catalonia (CEEISCAT), Agencia de Salut Publica de \\ Catalunya (ASPC), Generalitat de Catalunya, Badalona, Spain \\ ${ }^{2}$ The Institute for Health Science Research Germans Trias i Pujol (IGTP), Badalona, Spain \\ ${ }^{3}$ Department Paediatrics, Obstetrics and Gynaecology, and Preventive Medicine, Univ Autonoma de Barcelona, \\ Bellaterra, Spain \\ ${ }^{4}$ CIBER Epidemiologia y Salud Pública (CIBERESP), Madrid, Spain
}

Received 24 October 2016; Final revision 25 January 2017; Accepted 9 February 2017; first published online 8 March 2017

\section{SUMMARY}

Migrants are considered a key group at risk for HIV infection. This study describes differences between migrants and the Spanish-born population as they progress through the HIV care cascade in Catalonia, Spain. This study found that among people reached by prevention activities, migrants had a higher number of barriers to access HIV testing services than Spanishborn people, driven primarily by shared risk factors. Between 2001 and 2013, 9829 new HIV diagnoses were reported in Catalonia, the proportion of migrants increasing from 24\% in 2001 to $41 \%$ in 2013. Compared with Spanish-born people, migrants had a higher proportion of women at diagnosis $(24.6 \% v s .16 \cdot 7 \%)$, and were younger (median age of $33 v s .37$ ). The most frequent at-risk population was MSM (men who have sex with men) in both migrants and Spanish-born people, ( $40 \%$ and $43 \%$, respectively), although there were significant differences by region of origin. People from sub-Saharan Africa had the highest proportion of late diagnosis $(63 \cdot 7 \%)$. Compared with the Spanish-born population, migrants on follow-up had a lower proportion of people on antiretroviral therapy (ART) $(93.7 \%$ vs. $90.8 \%, P<0.001)$ and with viral suppression (87.2\% vs. $82.9 \%, P<0.001)$. Migrants have higher number of barriers to access HIV testing services, lower retention rates and proportions on ART as compared with Spanish-born people, these differences not being uniform between migrants from different regions.

Key words: Cascade, epidemiology, health services, HIV, information system, migrant.

\section{INTRODUCTION}

Population mobility has been identified as a key driver of the HIV epidemic [1], by both linking

\footnotetext{
* Author for correspondence: J. Reyes-Urueña, Centre for Epidemiological Studies on HIV/STI in Catalonia (CEEISCAT), Agencia de Salut Publica de Catalunya (ASPC), Generalitat de Catalunya, Badalona, Spain.

(Email: jmreyes@iconcologia.net)
}

geographically separate epidemics [1] and intensifying transmission through the introduction of risk behaviours that increase vulnerability to HIV infection [1-3]. Moreover, this can be exacerbated by other factors such as family fragmentation, adaptation to the host culture, language barriers, poor living conditions, job insecurity, unequal access to preventive, diagnostic and treatment services in host communities [4-6]. 
In 2014, migrants constituted a considerable proportion $(37 \%)$ of new HIV diagnoses in the EU/ EEA [7] and the evidence increasingly indicates that a high proportion of these acquired HIV after migration $[8,9]$. Although attention in recent years has focused on migrants from high prevalence countries with generalised epidemics, there is evidence that specific migrant groups from other regions with more concentrated epidemics, such as men who have sex with men (MSM) from Latin America, are also highly vulnerable to HIV [4, 10-12]. Late presentation for HIV diagnosis among migrants is also a key concern. A higher proportion of HIV-positive male and female migrants from low- and middle-income regions present late for HIV diagnosis as compared with native-born populations [10]. Migration has also been associated with slower progression through the HIV care cascade; with increased risk of nonadherence to antiretroviral therapy (ART), loss to follow-up, deterioration in CD4 count, HIV-related death and development of drug resistance [13].

Because of their increased vulnerability, migrants have been recognised as a priority group when designing programmes for HIV prevention and care in Europe $[10,14]$. To better understand the metrics and determinants that can improve the effectiveness of such programmes, this study aims to describe differences between migrants and the Spanish-born population at diagnosis and as they progress through the HIV care in Catalonia, Spain.

\section{MATERIALS AND METHODS}

We undertook descriptive and comparative data analysis to identify differences between migrants and Spanish-born people along the HIV care cascade, using multiple sources of information from the integrated Catalan sexually transmitted infection (STI)/ HIV Surveillance System (SIVES). Determinants of sexual risk and HIV test-seeking behaviours, HIV trends and clinical and epidemiological characteristics were all assessed. Migrants were defined as individuals born in a country other than Spain and aged 18 or over. Region of origin was determined by the country of birth and was classified as follows: Western Europe and North America, Eastern Europe, sub-Saharan Africa (SSA), North Africa, and Latin America and the Caribbean. The Spanish-born population was defined as people who reported their place of birth Spain. HIV and AIDS cases were defined according to the criteria of the European Centre for AIDS/
HIV Epidemiological Surveillance [15]. Retained in care was defined as the proportion enrolled in the PISCIS cohort during the study period and who had at least one visit in the year following after their enrolment. Late presentation was defined as a CD4 count of $<350$ cells/ $\mu 1$ or AIDS, and advanced HIV infection as a CD4 cell count below 200 cells/ml or AIDS at the time of HIV diagnosis, in line with the European Late Presenter Consensus Working Group [16]. Viral load suppression was defined as the proportion of patients on ART and who had $<50$ copies $/ \mathrm{ml}$ on the last measurement of the year. Loss to follow-up was defined as a patient who had been followed up at the HIV clinic, who had not had contact with the health facility for 1 year or more since their last recorded expected date of return or if there was one year or more between the expected date of return and the next clinic visit.

\section{Sources of data}

A full description of the information systems that form part of SIVES is available elsewhere [17]. This paper is structured in accordance with the conceptual depiction of the HIV cascade of services as described in WHO's Consolidated strategic information guidelines for HIV [18].

\section{People at risk and reached by prevention activities}

Sexual behaviour, risk practices for HIV transmission and knowledge of transmission routes were measured using data from behavioural monitoring studies conducted on people who inject drugs (PWID) [19], female sex workers (FSW) [20], MSM (from the ITACA cohort of HIV-negative MSM who attend a community centre [12], and the EMIS Project [21]). Information was available for different periods in each of the sources and data extraction was undertaken accordingly, collecting information on testing frequency, access to healthcare, drug consumption, condom use and knowledge about HIV transmission routes. This last item was built from responses given to questions about knowledge of HIV transmission. Data on testing activity from 2002 to 2014 were extracted from community-based voluntary counselling and testing services (CBVCT), which perform voluntary, free and anonymous HIV antibody testing [22]. The rapid HIV test used by CBVCT services is Determine HIV 1/2, Abbott Laboratories, Abbott Park, IL, USA. We calculated the total number of people tested and the number of reactive test results by risk group and region of origin. 


\section{People aware of their positive HIV status}

The primary data source was HIV and AIDS cases notified to the Catalan HIV Surveillance System from 2001 to 2013 [17]. We extracted information on the date of HIV diagnosis, socio-demographic characteristics, risk group and presentation with late and/or advanced HIV disease (including AIDS-defining conditions (ADC)), then described their trends.

\section{People enrolled in HIV care, on ART and with viral load suppressed}

Data used to estimate the proportion of people enrolled, on ART and virally suppressed in the HIV care cascade were derived from PISCIS cohort, which includes data on over $17000 \mathrm{HIV}$-positive people seen for follow-up in one of the participating centres in Catalonia and is more fully described elsewhere [23]. Each of these steps was analysed by socio-demographic characteristics and risk population in people under active follow-up. In Catalonia healthcare and access to treatment are universal and free of charge for all people including migrants with irregular legal status.

\section{Statistical analysis}

Differences in the distribution of demographic characteristics (sex, age, education), risk population (PWID, FSW and MSM), sex behaviour and clinical characteristics, were assessed by region of origin, using Pearson's $\chi^{2}$ test and Student's $t$ test for categorical and continuous variables, respectively. Comparisons were made to provide insight into which factors were linked to HIV infection by region of origin. Correlates were determined using a regression model and the odds ratios, along with $95 \%$ confidence intervals (CIs) calculated are reported. HIV prevalence was reported for PWID, FSW and MSM. Denominator populations used to calculate rates of new HIV diagnoses per 100000 population were derived from census data [24]. The ITACA cohort HIV incidence rate and corresponding 95\% CIs were calculated using person-time measures. In the absence of reliable estimates of the numbers of migrants (diagnosed and undiagnosed) living with HIV in Catalonia, we constructed a modified cascade starting with the people who were diagnosed and linked to care. Subsequent steps were calculated by applying to the preceding stage a proportion estimated from surveillance data and the proportions retained in care, on ART and virally suppressed were derived from the PISCIS cohort. A fuller description of the methods has been published elsewhere [25]. The level of significance was set at a $P$ value of $<0 \cdot 05$. Data analysis was performed using Stata 12. CollegeStation, TX: StataCorpLP.

\section{RESULTS}

\section{People at risk and reached by prevention activities}

Differences in HIV risk indicators by key population are shown in Table 1 stratified by migration status. Among PWID, the HIV prevalence was higher in Spanish-born people (35.9\%) compared with migrants $(22.4 \%)$ and a greater proportion of the Spanish-born population was knowledgeable about HIV transmission routes $(69 \cdot 7 \%$ vs. $77 \cdot 7 \%)$ and reported better access to substance abuse outpatient treatment centres $(69 \cdot 8 \% \mathrm{vs}$. $31 \cdot 0 \%)$, primary care $(72 \cdot 5 \% v s .49 \cdot 3 \%)$ and emergency departments $(35 \cdot 0 \%$ vs. $23 \cdot 4 \%)$. Among FSW, HIV prevalence was higher among the Spanish-born than migrants $(14 \cdot 7 \%$ vs. $0 \cdot 3 \%)$, Spanish-born FSW reporting higher proportions of illegal drug use (64.7\%) and inconsistent condom use with clients $(21 \cdot 9 \%)$. Migrant FSW reported having poorer access to healthcare services than Spanish-born FSW (65.6\% vs. 82.4\%). Latin America was the most frequent region of origin for MSM in both EMIS and the ITACA cohort (53.5\% and $57.9 \%$, respectively). In the ITACA cohort between 2009 and 2011, 3544 participants with at least one follow-up visit or those who had a first visit no longer than a year prior to the date of data censoring were included in the analysis contributing 3567.09 personyears (p-y) of follow-up and $85 \mathrm{MSM}$ seroconverted, giving an overall HIV incidence of 2.4 per 100 p-y $(95 \%$ CI 1.9-2.9) and ranging from $1 \cdot 21 / 100$ (2009) to $3 \cdot 1 / 100$ p-y (2011). The incidence was higher among migrant (3.7/100 p-y) than Spanish-born MSM (1.7/100 p-y), yet a greater proportion of migrant MSM were knowledgeable about HIV transmission routes than Spanishborn MSM and had also been tested more frequently.

CBVCTs have performed a total of 52378 tests since 2002, the annual number of tests rising from 810 in 2002 to 9454 in 2014 and the proportion of migrants increasing by $93.5 \%$ in the same period. Of the 18656 migrants $(35.6 \%)$ tested, $55.3 \%$ were men and the most frequent regions of origin were Latin America (44.7\%) and Western Europe (24.8\%). Migrants made up a majority of MSW (male sex workers; 55.7\%), PWID (78.8\%) and FSW (87.7\%). Test reactivity also varied by origin, with $3.9 \%(723 / 18656)$ of migrants 
Table 1. Comparison of HIV behavioural risk indicators between migrants and the Spanish-born population by key population, by follow-up periods for each information source, Catalonia, Spain

\begin{tabular}{|c|c|c|c|c|c|c|}
\hline Population & Information source & Period & Indicators & Migrants & $\begin{array}{l}\text { Spanish-born } \\
\text { population }\end{array}$ & $P$ value \\
\hline \multirow[t]{10}{*}{ PWID } & \multirow{10}{*}{$\begin{array}{l}\text { Bio-behavioural } \\
\text { monitoring }\end{array}$} & \multirow[t]{10}{*}{ 2012-2013 } & & $N=290$ & $N=440$ & \\
\hline & & & HIV prevalence $(\%)$ & $22 \cdot 4$ & $35 \cdot 9$ & $<0.001$ \\
\hline & & & $\begin{array}{l}\text { Tested for HIV in the last } 12 \text { months and knows the } \\
\text { test results }(\%)\end{array}$ & $72 \cdot 4$ & $69 \cdot 1$ & $0 \cdot 343$ \\
\hline & & & Access to health services (last 6 months) (\%) & & & \\
\hline & & & Substance abuse OTC & $31 \cdot 0$ & $69 \cdot 8$ & $<0.05$ \\
\hline & & & Primary care centre & $49 \cdot 3$ & $72 \cdot 5$ & $<0 \cdot 05$ \\
\hline & & & Emergency department (hospital) & $23 \cdot 4$ & $35 \cdot 0$ & $<0.05$ \\
\hline & & & $\begin{array}{l}\text { Condom use at last intercourse with a casual partner } \\
\text { (last } 6 \text { months) }(\%)\end{array}$ & $76 \cdot 8$ & $73 \cdot 1$ & $0 \cdot 48$ \\
\hline & & & Needle/syringe sharing in the last 6 months & $11 \cdot 8$ & $13 \cdot 3$ & 0.530 \\
\hline & & & Knowledge about routes of HIV transmission* (\%) & $69 \cdot 7$ & $77 \cdot 7$ & $0 \cdot 014$ \\
\hline \multirow[t]{8}{*}{ FSW } & \multirow{8}{*}{$\begin{array}{l}\text { Bio-behavioural } \\
\text { monitoring }\end{array}$} & \multirow[t]{8}{*}{2011} & & $N=365$ & $N=35$ & \\
\hline & & & HIV prevalence $(\%)$ & $0 \cdot 3$ & $14 \cdot 7$ & $<0 \cdot 001$ \\
\hline & & & $\begin{array}{l}\text { Tested for HIV in the last } 12 \text { months and knows the } \\
\text { test results }(\%)\end{array}$ & $66 \cdot 5$ & $82 \cdot 4$ & $0 \cdot 05$ \\
\hline & & & $\begin{array}{l}\text { Inconsistent condom use with a regular partner (last } \\
6 \text { months) }(\%)\end{array}$ & $95 \cdot 0$ & $88 \cdot 9$ & $0 \cdot 46$ \\
\hline & & & $\begin{array}{l}\text { Inconsistent condom use with clients (last } 6 \text { months) } \\
(\%)\end{array}$ & $8 \cdot 8$ & $21 \cdot 9$ & $<0 \cdot 05$ \\
\hline & & & Use of illegal drugs (at least once) & $28 \cdot 5$ & $64 \cdot 7$ & $<0 \cdot 001$ \\
\hline & & & Access to health services (last 6 months) (\%) & $65 \cdot 6$ & $82 \cdot 4$ & $0 \cdot 05$ \\
\hline & & & Knowledge about routes of HIV transmission $\dagger(\%)$ & $53 \cdot 5$ & $61 \cdot 8$ & $0 \cdot 35$ \\
\hline \multirow[t]{7}{*}{ MSM } & \multirow[t]{2}{*}{ ITACA cohort } & \multirow[t]{2}{*}{ 2009-2011 } & & $N=1731$ & $N=3336$ & \\
\hline & & & HIV incidence & $\begin{array}{l}3 \cdot 7 / 100 \\
\text { person-years CI } \\
95 \%(2 \cdot 7-4 \cdot 8)\end{array}$ & $\begin{array}{l}1 \cdot 7 / 100 \\
\text { person-years CI } \\
95 \%(1 \cdot 7-2 \cdot 2)\end{array}$ & - \\
\hline & \multirow[t]{5}{*}{ EMIS } & \multirow[t]{5}{*}{2010} & & $N=877$ & $N=2057$ & \\
\hline & & & $\begin{array}{l}\text { HIV self-reported prevalence (among those who } \\
\text { have been tested) }\end{array}$ & $15 \cdot 7$ & $10 \cdot 8$ & $<0.001$ \\
\hline & & & $\begin{array}{l}\text { Tested for HIV in the last } 12 \text { months and knows the } \\
\text { test results }(\%)\end{array}$ & $55 \cdot 8$ & $50 \cdot 3$ & $0 \cdot 01$ \\
\hline & & & Condom use at last intercourse (last 6 months) (\%) & $59 \cdot 7$ & $62 \cdot 4$ & $0 \cdot 20$ \\
\hline & & & Knowledge about routes of HIV transmissiont (\%) & 47 & $43 \cdot 5$ & $0 \cdot 08$ \\
\hline
\end{tabular}

PWID, people who inject drugs; MSM, men who have sex with men; FSW, female sex workers; OTC, outpatient treatment centres; CI, confidence interval.

* High knowledge was defined as correctly answering all of the following questions: (1) Can you reduce the risk of HIV transmission by having sex with one partner who has not contracted HIV and has no other partners? (2) Can the risk of HIV transmission be reduced by using a condom every time a sexual intercourse is performed? (3) Can a healthy-looking person have HIV? (4) Can you get HIV from a mosquito bite? (5) Can you get HIV by sharing a meal with an HIV-positive person?

$\uparrow$ Sufficient knowledge about routes of HIV transmission was considered if the individual answered in the affirmative that HIV is transmitted by the following three routes: (1) through used syringes, (2) by vaginal intercourse without a condom, (3) by anal intercourse without a condom.

+ High knowledge was considered by correctly answering the following items: (1) you cannot be sure that someone has HIV by their physical appearance; (2) effective HIV treatment reduces the risk of HIV transmission; (3) HIV is not transmitted by kissing, even with a deep kiss, because saliva does not transmit the virus; (4) you can get HIV through your penis while practicing unprotected anal or vaginal active penetration (penetrate) with an infected partner, even without ejaculating; (5) you can get HIV through your anus during unprotected anal intercourse in passive penetration (being penetrated) with an infected partner. 


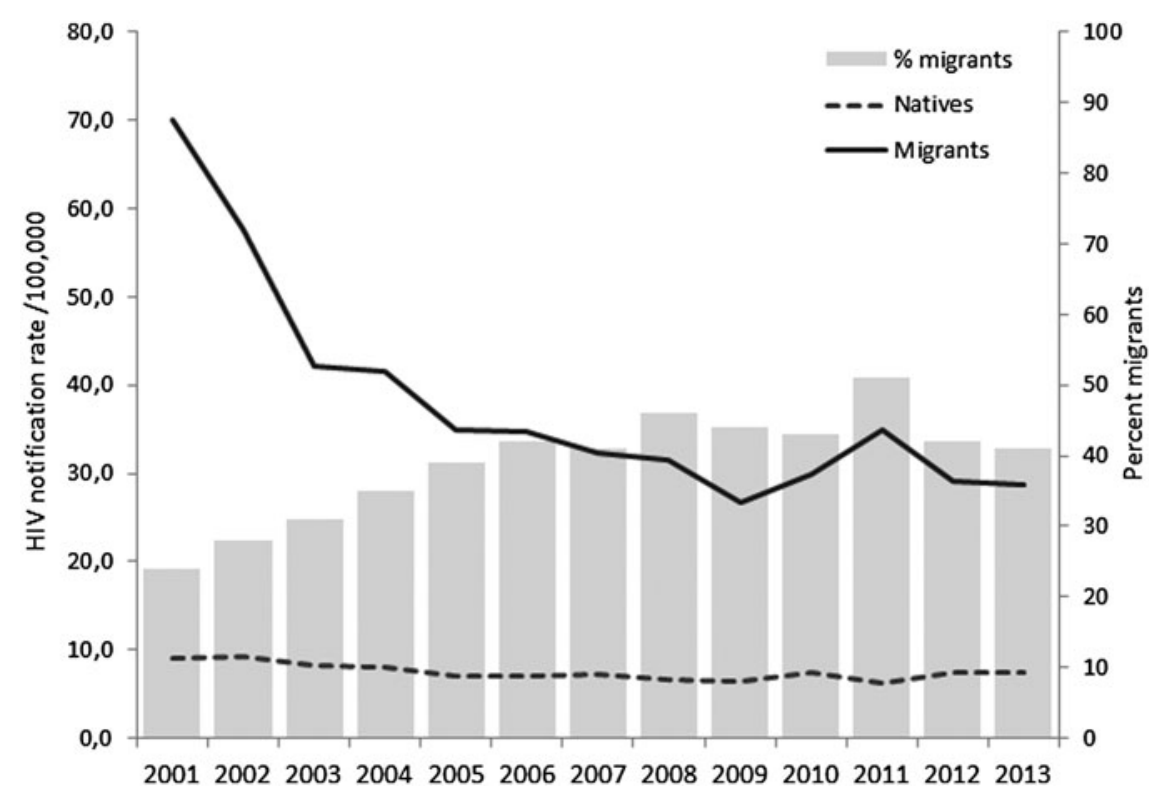

Fig. 1. Trend in HIV notification rates (per 100000 inhabitants) in Spanish-born population and migrants and per cent of migrants among new notifications. Catalonia - Spain, 2001-2013.

having a reactive test as compared with $2 \cdot 0 \%(636 / 31$ 162) of the Spanish-born population.

\section{People aware of their positive HIV status}

Between 2001 and 2013, 9829 new HIV diagnoses were reported to the Catalan HIV surveillance system, $38.5 \%$ of whom were migrants. The HIV notification rate in Spanish-born people has remained stable throughout, with a median notification rate of $7 \cdot 4$ cases per 100000 population. However, the proportion of migrants among notified HIV cases has increased progressively from $24 \%$ in 2001 to $41 \%$ in 2013 and although it has declined since 2011, the HIV notification rate among migrants was five times higher than that of the Spanish-born population in 2013 (Fig. 1). The epidemiological profile of migrants was different to that of the Spanish-born population (Table 2); there was a higher proportion of women $(24.6 \%$ and $16.7 \%$, respectively) and they were younger at diagnosis (median age of $33 v s .37$, respectively). In both migrants and Spanish-born people, the most frequent at-risk population was MSM ( $40 \%$ and $43 \%$, respectively) and there were significant differences by region of origin. Whereas in people from Western Europe and Latin America the main risk population was MSM (65\% and $60 \cdot 4 \%$, respectively), in people from Eastern Europe it was PWID (34.5\%) and in those from SSA it was HTS (heterosexuals) in both men $(46 \cdot 3 \%)$ and women $(37 \cdot 6 \%)$. In the
Spanish-born population, the proportion of late diagnoses was lower $(50 \cdot 4 \%)$ than in migrants $(54 \cdot 0 \%)$. People from SSA had the highest proportion of late diagnosis $(63 \cdot 7 \%)$ and advanced disease (43.4\%). There were no significant differences in the proportion of ADC between migrants and natives, but the pattern of ADC did vary between them. The most common ADC among the Spanish-born population was Pneumocystis jirovecii pneumonia $(30 \cdot 9 \%)$, whereas among migrants it was tuberculosis $(30 \cdot 1 \%)$.

\section{People enrolled in HIV care, on ART and with viral load suppressed}

Table 3 shows the socio-demographic and clinical differences seen between migrants and the Spanish-born population under follow-up. At the end of 2012 there were 7248 HIV-positive patients under active follow-up in the PISCIS cohort, $6022(83 \%)$ of whom had a known country of origin. Migrants accounted for $1828(30 \cdot 4 \%)$ of these patients, $53 \cdot 6 \%$ (956) originating from Latin America or the Caribbean. As at enrolment, most people under follow-up were men $(80 \cdot 2 \%)$, the largest transmission group being MSM (42.6\%). MSM made up $76.0 \%$ and $68.7 \%$ of migrants from Western Europe and Latin America/Caribbean, respectively, but only $7 \%$ of those from Africa.

Median time under follow-up was 5.5 years in Spanish-born people and 3.5 years in migrants. The longest follow-up time was for Africans (4.4 years) 
Table 2. Baseline epidemiological characteristics of people aware of their HIV-positive status and notified to the surveillance system, by origin and by geographical region (Catalonia, 2001-2013)

\begin{tabular}{|c|c|c|c|c|c|c|c|c|c|c|c|c|c|}
\hline & $\begin{array}{l}\text { Total } \\
n(\%)\end{array}$ & $\begin{array}{l}\text { Spanish-born } \\
\text { population } \\
n(\%)\end{array}$ & $\begin{array}{l}\text { Migrants } \\
n(\%)\end{array}$ & $P$ value* & OR & CI $95 \%$ & & $\begin{array}{l}\text { WE } \\
n(\%) !\end{array}$ & $\begin{array}{l}\mathrm{EE} \\
n(\%) !\end{array}$ & $\begin{array}{l}\text { LAC } \\
n(\%):\end{array}$ & $\begin{array}{l}\text { SSA } \\
n(\%) !\end{array}$ & $\begin{array}{l}\text { NA } \\
n(\%)\end{array}$ & $P$ value $\dagger$ \\
\hline HIV cases & $9829(100)$ & $5969(59 \cdot 6)$ & $3860(38 \cdot 5)$ & $<0 \cdot 001$ & & & & $469(12 \cdot 2)$ & $284(7 \cdot 4)$ & $1802(46 \cdot 7)$ & $951(24 \cdot 6)$ & $189(4 \cdot 9)$ & $<0 \cdot 001$ \\
\hline Sex & & & & $<0 \cdot 001$ & & & & & & & & & $<0 \cdot 001$ \\
\hline Men & $7879(80 \cdot 2)$ & $4970(83 \cdot 3)$ & $2909(75 \cdot 4)$ & & $1 \cdot 0$ & & & $434(92 \cdot 5)$ & $184(64 \cdot 8)$ & $1430(79 \cdot 4)$ & $548(57 \cdot 6)$ & $158(83 \cdot 6)$ & \\
\hline Women & $1950(19 \cdot 8)$ & $999(16 \cdot 7)$ & $951(24 \cdot 6)$ & & 1.6 & $1.5-1.8$ & & $35(7 \cdot 5)$ & $100(35 \cdot 2)$ & $372(20 \cdot 6)$ & $403(42 \cdot 4)$ & $31(16 \cdot 4)$ & \\
\hline Age (median) & 35 & 37 & 33 & $<0 \cdot 001$ & & & & & & & & & \\
\hline Age group & & & & $<0.001$ & & & & & & & & & $<0 \cdot 001$ \\
\hline$<20$ & $155(1 \cdot 6)$ & $78(1 \cdot 3)$ & $77(2 \cdot 0)$ & & 0.8 & $0 \cdot 6-1 \cdot 2$ & & $1(0 \cdot 2)$ & $9(3 \cdot 2)$ & $38(2 \cdot 1)$ & $28(2 \cdot 5)$ & $3(1 \cdot 6)$ & \\
\hline $20-39$ & $6426(65 \cdot 4)$ & $3517(58 \cdot 9)$ & $2909(75 \cdot 3)$ & & $1 \cdot 0$ & & & $325(69 \cdot 1)$ & $249(87 \cdot 7)$ & $1379(76 \cdot 5)$ & $694(73 \cdot 0)$ & $143(75 \cdot 7)$ & \\
\hline $40-49$ & $2081(21 \cdot 2)$ & $1414(23 \cdot 7)$ & $667(17 \cdot 3)$ & & 0.5 & $0 \cdot 3-0 \cdot 7$ & & $115(245)$ & $19(6 \cdot 7)$ & $301(167)$ & $167(17 \cdot 6)$ & $33(17 \cdot 5)$ & \\
\hline$\geqslant 50$ & $1168(11.9)$ & $960(16 \cdot 10)$ & $207(5 \cdot 4)$ & & $0 \cdot 2$ & $0 \cdot 2-0 \cdot 3$ & & $29(6 \cdot 2)$ & $7(2 \cdot 5)$ & $84(4 \cdot 7)$ & $65(6 \cdot 8)$ & $10(5 \cdot 3)$ & \\
\hline Risk population & & & & $<0 \cdot 001$ & & & & & & & & & $<0 \cdot 001$ \\
\hline PWID & $1225(12 \cdot 5)$ & $964(16 \cdot 2)$ & $261(6 \cdot 8)$ & & $2 \cdot 7$ & $2 \cdot 3-3 \cdot 2$ & & $58(12 \cdot 4)$ & $98(34 \cdot 5)$ & $22(1 \cdot 2)$ & $35(18 \cdot 5)$ & $35(18 \cdot 5)$ & \\
\hline Heterosexual men & $\begin{array}{c}20638 \\
(21 \cdot 0)\end{array}$ & $1183(19 \cdot 8)$ & $880(22 \cdot 8)$ & & $1 \cdot 0$ & & & $42(9 \cdot 0)$ & $31(10 \cdot 9)$ & $251(13 \cdot 9)$ & $440(46 \cdot 3)$ & $80(42 \cdot 3)$ & \\
\hline MSM & $4113(41 \cdot 8)$ & $2569(43 \cdot 0)$ & $1544(40 \cdot 0)$ & & $2 \cdot 2$ & $1 \cdot 9-2 \cdot 6$ & & $305(65 \cdot 0)$ & $49(17 \cdot 3)$ & $1088(60 \cdot 4)$ & $15(1 \cdot 6)$ & $24(12 \cdot 7)$ & \\
\hline Heterosexual women & $1530(15 \cdot 6)$ & $723(12 \cdot 1)$ & $807(20 \cdot 9)$ & & $4 \cdot 1$ & $3 \cdot 5-4 \cdot 9$ & 产 & $22(4 \cdot 7)$ & $65(22 \cdot 9)$ & $326(18 \cdot 1)$ & $358(37 \cdot 6)$ & $29(15 \cdot 3)$ & \\
\hline Unknown & $899(9 \cdot 1)$ & $530(8 \cdot 9)$ & $368(9 \cdot 5)$ & & $2 \cdot 6$ & $2 \cdot 1-3 \cdot 1$ & 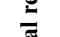 & $42(9 \cdot 0)$ & $40(14 \cdot 1)$ & $115(6 \cdot 4)$ & $126(13 \cdot 2)$ & $21(11 \cdot 1)$ & \\
\hline Late diagnosis§ & $4269(51 \cdot 8)$ & $2541(50 \cdot 4)$ & $1728(54 \cdot 0)$ & $<0.001$ & $1 \cdot 2$ & $1 \cdot 1-1 \cdot 1$ & 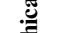 & $176(44 \cdot 3)$ & $113(48 \cdot 9)$ & $790(52 \cdot 0)$ & $494(63 \cdot 7)$ & $77(50 \cdot 3)$ & $<0.001$ \\
\hline Advanced HIV & $2714(32.9)$ & $1662(32 \cdot 9)$ & $1052(32 \cdot 9)$ & 0.933 & $1 \cdot 0$ & $0 \cdot 9-1 \cdot 1$ & $\overline{\bar{\pi}}$ & $91(22 \cdot 9)$ & $66(28 \cdot 6)$ & $455(30 \cdot 0)$ & $336(43 \cdot 4)$ & $52(32 \cdot 9)$ & $<0 \cdot 001$ \\
\hline AIDS-indicator conditions & $1859(18.9)$ & $1152(19 \cdot 3)$ & $707(18 \cdot 3)$ & $0 \cdot 222$ & 0.9 & $0 \cdot 8-1 \cdot 0$ & 50 & $64(13 \cdot 6)$ & $29(10 \cdot 2)$ & $298(16 \cdot 5)$ & $189(19 \cdot 9)$ & $35(18 \cdot 5)$ & $<0 \cdot 001$ \\
\hline $\begin{array}{l}\text { Pneumocystis jirovecii } \\
\text { pneumonia }\end{array}$ & $457(26 \cdot 3)$ & $335(30 \cdot 9)$ & $122(18 \cdot 8)$ & & & & छัँ & $18(28 \cdot 1)$ & $4(13 \cdot 8)$ & $58(19 \cdot 5)$ & $23(12 \cdot 2)$ & $10(28 \cdot 6)$ & \\
\hline $\begin{array}{l}\text { Mycobacterium } \\
\text { tuberculosis - pulmonary, } \\
\text { extrapulmonary or miliary }\end{array}$ & $364(21 \cdot 0)$ & $168(15 \cdot 4)$ & $196(30 \cdot 1)$ & & & & & $10(15 \cdot 7)$ & $13(44 \cdot 8)$ & $66(22 \cdot 1)$ & $88(22 \cdot 8)$ & $8(15 \cdot 6)$ & \\
\hline $\begin{array}{l}\text { Esophageal } \\
\text { candidiasis }\end{array}$ & $192(11 \cdot 1)$ & $137(12 \cdot 6)$ & $55(8 \cdot 5)$ & & & & & $12(18 \cdot 8)$ & $2(6 \cdot 9)$ & $25(8 \cdot 4)$ & $7(3 \cdot 7)$ & $5(14 \cdot 3)$ & \\
\hline Kaposi sarcoma & $164(9 \cdot 5)$ & $107(9 \cdot 9)$ & $57(8 \cdot 8)$ & & & & & $5(7 \cdot 8)$ & $1(3 \cdot 4)$ & $35(11 \cdot 7)$ & $10(5 \cdot 3)$ & $4(11 \cdot 4)$ & \\
\hline $\begin{array}{l}\text { Toxoplasmosis of } \\
\text { brain }\end{array}$ & $118(6 \cdot 8)$ & $63(5 \cdot 8)$ & $55(8 \cdot 5)$ & & & & & $3(4 \cdot 7)$ & $3(10 \cdot 3)$ & $26(8 \cdot 7)$ & $19(10 \cdot 1)$ & $3(8 \cdot 6)$ & \\
\hline Linfoma no Hodgkin & $71(4 \cdot 1)$ & $57(5 \cdot 3)$ & $14(2 \cdot 2)$ & & & & & $0(0 \cdot 0)$ & $0(0 \cdot 0)$ & $11(3 \cdot 7)$ & $1(0 \cdot 5)$ & $1(2 \cdot 9)$ & \\
\hline Wasting syndrome caused by HIV & $87(5 \cdot 0)$ & $55(5 \cdot 1)$ & $32(4 \cdot 9)$ & & & & & $5(7 \cdot 8)$ & $1(3 \cdot 4)$ & $8(2 \cdot 7)$ & $16(8 \cdot 5)$ & $1(2 \cdot 9)$ & \\
\hline Others & $406(21 \cdot 8)$ & $565(49 \cdot 0)$ & $176(24 \cdot 9)$ & & & & & $11(17 \cdot 1)$ & $5(17 \cdot 2)$ & $69(23 \cdot 2)$ & $25(13 \cdot 2)$ & $3(0 \cdot 3)$ & \\
\hline
\end{tabular}

PWID, people who inject drugs; MSM, men who have sex with men; WE, Western Europe; EE, Eastern Europe; LAC, Latin America and the Caribbean; SSA, sub-Saharan Africa; NA, North Africa; OR, odds ratio; CI, confidence interval.

$* \chi^{2}$ test.

$\dagger \chi^{2}$ test comparing migrants by region of origin with Spanish-born population.

$\$$ Percentages calculated using as a denominator the total number of migrants newly diagnosed with HIV, Catalonia, 2001-2013, except in the case of late and advanced diagnosis (see notes $\S$ and $\uparrow$ ).

$\S$ Due to missing CD4 count data, the denominators for late and advanced diagnosis are: 5045 in natives, 3202 in migrants and 8247 in total.

I Due to missing CD4 count data, the denominators for region of origin are: 397 in WE, 231 in EE, 1518 in LAC, 775 in SSA and 153 in NA. 
Table 3. Comparison of socio-demographic characteristics and clinical features of people enrolled in HIV care, by place and region of origin, Catalonia-Spain, $1998-2012$.

\begin{tabular}{|c|c|c|c|c|c|c|c|c|c|c|}
\hline & $\begin{array}{l}\text { Spanish-born } \\
\text { population } \\
n(\%)\end{array}$ & $\begin{array}{l}\text { Migrants } \\
n(\%)\end{array}$ & $\begin{array}{l}\text { Total } \\
n(\%)\end{array}$ & $P$ value $\dagger$ & & $\begin{array}{l}\text { WE } \\
n(\%) \$\end{array}$ & $\begin{array}{l}\mathrm{EE} \\
n(\%)\end{array}$ & $\begin{array}{l}\text { LAC } \\
n(\%) \$\end{array}$ & $\begin{array}{l}\text { SSA and } \\
\text { NA } \\
n(\%)\end{array}$ & $\begin{array}{l}\text { Others } \\
n(\%) \ddagger\end{array}$ \\
\hline Total & $4194(69 \cdot 6)$ & $1828(30 \cdot 4)$ & $6022(100)$ & & & $316(17 \cdot 3)$ & $95(5 \cdot 2)$ & $980(53 \cdot 6)$ & $271(14 \cdot 8)$ & $166(9 \cdot 1)$ \\
\hline \multicolumn{11}{|l|}{ Sex } \\
\hline Men & $3363(80 \cdot 2)$ & $1466(80 \cdot 2)$ & $4829(80 \cdot 2)$ & $1 \cdot 000$ & & $293(92 \cdot 7)$ & $60(63 \cdot 2)$ & $809(82 \cdot 6)$ & $173(63 \cdot 8)$ & $131(78 \cdot 9)$ \\
\hline Women & $831(19 \cdot 8)$ & $362(19 \cdot 8)$ & $1193(19 \cdot 8)$ & & & $23(7 \cdot 3)$ & $35(36 \cdot 8)$ & $171(17 \cdot 4)$ & $98(36 \cdot 2)$ & $35(21 \cdot 1)$ \\
\hline Age median (IQR) & $37(31-44)$ & $34(29-40)$ & $36(30-43)$ & $<0 \cdot 001 \S$ & & $36(31-41)$ & $31(26-36)$ & $34(29-40)$ & $36(29-41)$ & $36(30-41)$ \\
\hline \multicolumn{11}{|l|}{ Risk population* } \\
\hline PWID & $1034(24 \cdot 7)$ & $87(4 \cdot 8)$ & $1121(18 \cdot 6)$ & $<0.001$ & \multirow{5}{*}{ 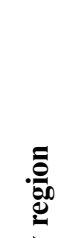 } & $26(8 \cdot 2)$ & $19(20 \cdot 0)$ & $20(2 \cdot 0)$ & $11(4 \cdot 1)$ & $11(6 \cdot 6)$ \\
\hline MSM & $1786(42 \cdot 6)$ & $1026(56 \cdot 1)$ & $2812(46 \cdot 7)$ & & & $240(76 \cdot 0)$ & $31(32 \cdot 6)$ & $673(68 \cdot 7)$ & $19(7 \cdot 0)$ & $63(38 \cdot 0)$ \\
\hline $\begin{array}{l}\text { Heterosexual women } \\
\text { and men }\end{array}$ & $1075(25 \cdot 6)$ & $624(34 \cdot 1)$ & $1699(28 \cdot 2)$ & & & $37(11 \cdot 7)$ & $32(33 \cdot 7)$ & $259(26 \cdot 4)$ & $218(80 \cdot 4)$ & $78(47 \cdot 0)$ \\
\hline Others & $297(7 \cdot 1)$ & $91(5 \cdot 0)$ & $388(6 \cdot 4)$ & & & $13(4 \cdot 1)$ & $13(14 \cdot 3)$ & $28(2 \cdot 9)$ & $23(8 \cdot 5)$ & $14(8 \cdot 4)$ \\
\hline \multicolumn{10}{|l|}{ Educational level } & \\
\hline $\begin{array}{l}\text { Primary education or } \\
\text { less }\end{array}$ & $1215(29 \cdot 0)$ & $435(23 \cdot 8)$ & $1650(27 \cdot 4)$ & $<0.001$ & $\stackrel{\vec{b}}{\leftrightarrows}$ & $39(12 \cdot 3)$ & $30(31 \cdot 6)$ & $184(18 \cdot 8)$ & $124(45 \cdot 8)$ & $58(34 \cdot 9)$ \\
\hline Secondary education & $795(19 \cdot 0)$ & $571(31 \cdot 2)$ & $1366(22 \cdot 7)$ & & $\overline{\bar{T}_{0}}$ & $84(26 \cdot 6)$ & $30(31 \cdot 6)$ & $352(35.92)$ & $49(18 \cdot 1)$ & $56(33 \cdot 7)$ \\
\hline $\begin{array}{l}\text { Undergraduate } \\
\text { education }\end{array}$ & $491(11 \cdot 7)$ & $412(22 \cdot 5)$ & $903(15 \cdot 0)$ & & $\sum^{200}$ & $120(38 \cdot 0)$ & $16(16 \cdot 9)$ & $235(24 \cdot 0)$ & $14(5 \cdot 28)$ & $27(16 \cdot 3)$ \\
\hline Unknown & $1693(40 \cdot 4)$ & $410(22 \cdot 4)$ & $2103(34 \cdot 9)$ & & & $73(23 \cdot 1)$ & $14(20 \cdot 0)$ & $209(21 \cdot 3)$ & $84(31 \cdot 0)$ & $25(15 \cdot 1)$ \\
\hline $\begin{array}{l}\text { Years on follow up median } \\
\text { (IQR) }\end{array}$ & $5 \cdot 5(2 \cdot 7-9 \cdot 4)$ & $3 \cdot 5(1 \cdot 5-6 \cdot 2)$ & $5 \cdot 4(2 \cdot 5-9 \cdot 5)$ & $<0 \cdot 001 \S$ & & $3 \cdot 4(1 \cdot 4-6 \cdot 4)$ & $3 \cdot 1(1 \cdot 7-6 \cdot 2)$ & $3 \cdot 5(1 \cdot 5-5 \cdot 9)$ & $4 \cdot 4(1 \cdot 8-7 \cdot 6)$ & $2 \cdot 6(1 \cdot 2-4 \cdot 5)$ \\
\hline Number of people on ART & $3931(93 \cdot 7)$ & $1659(90 \cdot 8)$ & $5590(92 \cdot 8)$ & $<0 \cdot 001$ & & $290(91 \cdot 8)$ & $86(90 \cdot 5)$ & $893(91 \cdot 1)$ & $256(94 \cdot 5)$ & $134(80 \cdot 7)$ \\
\hline $\begin{array}{l}\text { Number of people with } \\
\text { suppressed VLף }\end{array}$ & $3655(87 \cdot 2)$ & $1515(82 \cdot 9)$ & $5170(85 \cdot 8)$ & $<0.001$ & & $268(84 \cdot 8)$ & $78(82 \cdot 1)$ & $816(83 \cdot 3)$ & $230(84 \cdot 9)$ & $123(74 \cdot 1)$ \\
\hline
\end{tabular}

PWID, people who inject drugs; MSM, men who have sex with men; ART, antiretroviral therapy; WE, Western Europe; EE, Eastern Europe; LAC, Latin America and the Caribbean; SSA and NA, sub-Saharan Africa and North Africa; IQR, interquartile range; VL, viral load.

* Only two had an unknown risk group.

$\dagger \chi^{2}$ test.

$\$$ Percentages were calculated using as a denominator the total number of migrants from the PISCIS cohort, except in the case of AIDS-defining event where the denominator was the total number of people who had an indicator condition at enrolment in the PISCIS cohort.

$\S$ Student's $t$ test.

9 Percentages were calculated using as a denominator the total number of new HIV diagnosis at enrolment in the PISCIS cohort. 

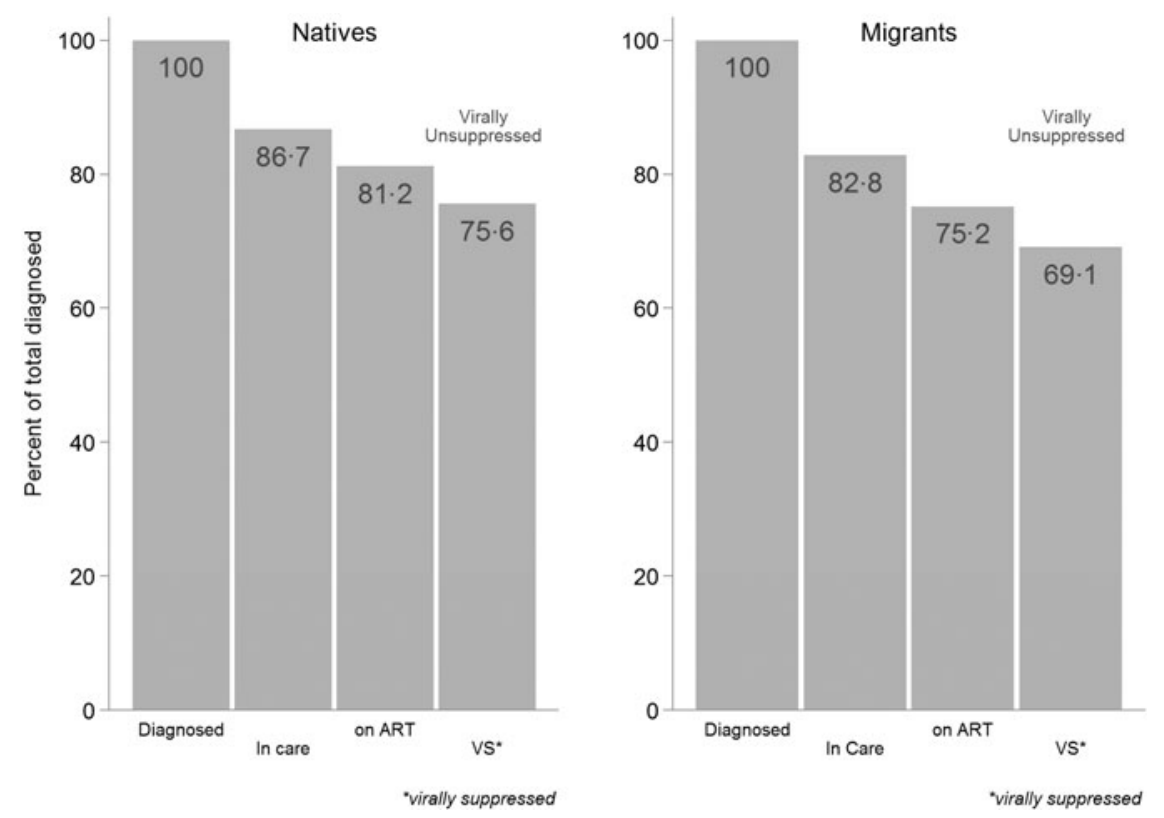

Fig. 2. HIV healthcare cascade comparing the Spanish-born population with migrants, Catalonia - Spain.

and the shortest for Eastern Europeans (3.1 years). Clinical follow-up 1 year after diagnosis was lower for migrants $(82.8 \%)$ than for Spanish-born people $(87 \cdot 6 \%)$. Of those on follow-up and with known migration status, $93.7 \%$ of Spanish-born people were on ART as compared with $90.8 \%$ of migrants $(P<0 \cdot 001)$. The proportion on ART varied by region of origin, though not reaching significance.

Data on viral suppression at the last visit was available for 5384/5477 patients on ART and with known migration status. A higher proportion of patients under follow-up with viral suppression were Spanish-born as compared with migrants $(87 \cdot 2 \%$ vs. $82.9 \%, P<0 \cdot 001)$. Viral suppression was highest in Africans $(84.9 \%)$ and Western Europeans (84.8\%) and lowest in Eastern Europeans $(82 \cdot 1 \%)$ and others $(74 \cdot 1 \%)$. Figure 2 shows the differences in the HIV care cascade by origin. As a percentage of the total under follow-up, migrants experience greater losses in retention in care $(83 \%$ vs. $88 \%$ retention, respectively) and in the proportion on ART (93.7\% vs. $90 \cdot 8 \%)$ and virally suppressed $(82.9 \%$ vs. $87 \cdot 2 \%)$. When stratified by region of origin, the difference in the proportion on ART is greatest for the region 'Others' (80.7\%) and Eastern Europeans (90.5\%). The sum of these differences between migrants and Spanish-born people along the cascade means that only $67 \%$ of migrants diagnosed with HIV had viral suppression, $8 \%$ lower than the Spanish-born at $75 \%$.

\section{DISCUSSION}

These are the first analyses to use multiple data sources to describe the differences between migrants and the Spanish-born population as they progress through the cascade of HIV prevention and care in Catalonia, Spain from notification to viral suppression. We show that disparities in health are evident between migrants and their native counterparts, with migrants having higher number of barriers to access HIV testing services as well as lower retention rates and proportions on ART, but it is worth noting that along the care cascade, variation between migrants form different regions is greater than that between migrants and the Spanish-born population.

\section{People at risk and reached by prevention activities}

This study found that among people reached by prevention activities, migrants experienced a higher number of barriers to access HIV testing services than the Spanish-born population and that these needs are driven primarily by shared risk factors. Our results showed that access to HIV testing differs by subpopulation, results which are in line with the HIV testing trends for Spain as a whole [26]. The general trend in Spain towards increased testing is not uniform across most-at-risk populations and actually decreases in some groups, such as FSW and PWID [26]. Lower HIV testing uptake in migrants who are PWID can be 
explained by poor access to drug treatment centres [3] and healthcare [27], whereas among FSW this reduction may be part of a broader trend throughout Europe of worsening access to healthcare and an observed shift in the working environment from outdoors to indoors that hampers sex worker access to prevention programmes $[28,29]$. Our research shows that migrant PWID and FSW reported health protective behaviours, but there is evidence that, over time, migrants tend to acquire the practices of their localborn counterparts; migrant FSW may start to consume illicit drugs [28], whereas migrants who are PWID may increase their consumption or adopt local consumption patterns [3]. Migrant MSM may engage in higher risk behaviours than Spanish-born MSM, such as condomless anal sex $[30,31]$. Consistent with this, migrant MSM, especially those born in Latin America, have high HIV prevalence rates among those diagnosed with syphilis and gonorrhoea, as well as high prevalence and incidence rates of STIs in those newly diagnosed with HIV [30]. Because migrant MSM perceive themselves as being at high risk for HIV, they are more likely to have ever been tested for HIV than the native MSM [32, 33], an observation supported by our data, although untested migrant MSM are particularly hard to reach [32]. As has been described, members of key populations who are also migrants are an important risk group for HIV, highlighting a need to design socially and culturally heterogeneous primary prevention programmes that address the needs of these sub-populations. These interventions should be designed to integrate gender perspective into sexual and reproductive health programmes specially directed at Latin American MSM, and bring access to HIV testing as close to the community as possible.

\section{People aware of their positive HIV status}

The epidemiological characteristics of migrants aware of their HIV status were similar to that of the Spanish-born population, being mainly young and MSM. However, consistent with data published from the rest of Spain, migrants are not a homogeneous group, their epidemiological characteristics reflecting those of the country of origin [34, 35]. In Europe, recent data show that nearly two-fifths of all HIV cases reported between 2007 and 2012 were migrants [10]. Although the biggest group of newly diagnosed migrants is still from SSA, the proportion is falling, especially in women [10]. At the same time, HIV diagnoses in people who have moved from one European country to another or from Latin America, Asia and the Middle East have continued to rise. Many of those born in the latter countries are MSM. Across Europe, there are now as many HIV diagnoses in migrant MSM as in migrant African women [10], indicating that the HIV European epidemic is now concentrated in MSM, with many MSM coming from Latin America. In our study, newly diagnosed migrants were more likely to present late $(54.0 \%)$, especially in people from SSA. These findings concord with previous studies from Spain and in Europe that identify being a migrant as a risk factor for late HIV diagnosis and presentation for care $[10,36,37]$. In Europe, people born in African, Asian and Latin American countries are the most likely to be diagnosed late with HIV [10]. Recently, European countries have made it increasingly difficult for migrants to access healthcare and this may negatively affect the late diagnosis rate [38]. Other barriers are related to the limited capacity of health services and providers to meet the specific needs of migrants, further limiting their access [27]. In the case of Spain, a country with mixed or universal healthcare systems, the main barriers are structural and organisational, such as inconsistent referral and coordination between service tiers [39]. In addition, timely entry into care may be hampered by poor understanding of administrative procedures and/or language barriers [13]. In order to help tackle all these barriers and ensure smooth progress along the HIV care continuum, health systems need to reduce differential access to care between migrants and Spanish-born people by designing HIV care programmes that take into consideration barriers due to culture, language and stigma. These programmes should include biomedical, behavioural and structural interventions that address the complex interplay of the underlying determinants of HIV transmission and would need to be implemented in the medium-to-long term to maximise their impact.

\section{People enrolled in HIV care on antiretroviral ART and with viral load suppression}

As described elsewhere in Europe and Spain [11, 13, $37,40]$, this study also shows that a lower proportion of migrants are enrolled in HIV care, on ART and have viral suppression, as compared with the Spanishborn population. Explanations include unfamiliarity with the host nation, miscommunication with HIV service providers and inadequate knowledge of patient 
rights and obligations [13]. Notwithstanding these discussions in the literature, our findings would suggest that in Catalonia at least, some of the differences between migrants from different regions (and by extension between migrants and the Spanish-born population) may be explained by differential patterns of migration rather than only differential access. Together with national statistical estimates of changing patterns of migration [24], our data provide evidence for this in the median duration of follow-up; Africans have the longest duration of follow-up because immigration from SSA has fallen off markedly since 2008 restricting the numbers of new SSA migrants, lengthening the median follow-up of those that remained. As a consequence, the probability of having been treated with ART increases. Conversely, those with the shortest median follow-up also have the lowest proportions on ART because they have had less time to be started on ART. Furthermore, the health-seeking behaviours of migrants are not always fully understood by service providers [13]. In Switzerland, higher rates of loss to follow-up among migrants have been explained by legal status or deportations, individuals returning to their home countries, higher geographical mobility and barriers to regularly accessing services [41]. Several of these barriers have also been associated with poor viral suppression, and are further exacerbated by the high mobility of individuals [13]. In order to facilitate uninterrupted access to treatment and provide realistic estimates for each step of the cascade, it is important that migrants are able to easily receive their HIV treatment at different facilities without being lost. People with diagnosed HIV, irrespective of their migration status can be better tracked across services and facilities where population-based cohorts are used or dynamic centralised registers set up [13]. In Catalonia, there is still a substantial gap in the way migrants' demographic and health information is collected and any improvements could also provide an opportunity to identify hidden disparities or inequalities in migrant's access to healthcare and better understand the determinants associated with loss to follow-up at successive stages of the cascade of HIV prevention and care in Catalonia.

Our study does have some limitations. The data are incomplete and come from different sources using different methods, making it difficult to generalise. Furthermore, available information is partial and not representative of all the different subpopulations of migrants across Catalonia. Despite this limitation, no other study has used so many different sources of information to describe the differences between migrants and the Spanish-born population as they progress through the cascade of HIV prevention and care. An important limitation is that we have not yet been able to study the differences in rates of undiagnosed HIV between migrants and the Spanish-born population. The various subgroup analyses completed for this study may not necessarily explain complex differences in global HIV epidemic dynamics among migrants, but they do demonstrate that high HIV prevalence rates among migrants are heterogeneous.

In conclusion, disparities in health are evident between migrants and their Spanish-born counterparts, with migrants having a higher number of barriers to access HIV testing services as well as lower retention rates and proportions on ART. Similar differences are also observed between migrants form different regions. Effective HIV testing and treatment programmes for migrants should be integrated into a balanced combination prevention framework that includes biomedical, behavioural and structural interventions that are stable in time and address the complex interplay of underlying determinants of HIV transmission and are especially tailored to migrant needs. All this requires unrestricted access to a continuum of HIV prevention, testing and care services, implemented through a multi-sectorial and participatory approach that takes into consideration the political, economic and social contexts.

\section{ACKNOWLEDGEMENTS}

The authors gratitude goes to all the Doctors who notified the HIV cases and to the HIV units which are treating patients in Catalonia - Spain, and also to all the professionals working in CBVCT services in Catalonia. This study received no specific grant from any funding agency.

\section{DECLARATION OF INTEREST}

None.

\section{REFERENCES}

1. Deane KD, Parkhurst JO, Johnston D. Linking migration, mobility and HIV. Tropical Medicine \& International Health 2010; 15: 1458-1463.

2. Coffee M, Lurie MN, Garnett GP. Modelling the impact of migration on the HIV epidemic in South Africa. AIDS 2007; 21: 343-350.

3. Saigí N, Espelt A, Folch C, et al. Differences in illegal drug consumption between native and immigrants in a 
large sample of injected drug users in Catalonia (Spain). Adicciones 2014; 26: 69-76.

4. Joint United Nations Programme on HIVIAIDS (UNAIDS). The gap report UNAIDS [Internet]. Geneva, Switzerland; 2014. http://www.unaids.org/sites/ default/files/media_asset/UNAIDS_Gap_report_en.pdf.

5. Weine SM, Kashuba AB. Labor migration and HIV risk: a systematic review of the literature. AIDS and Behavior 2012; 16: 1605-1621.

6. European Centre for Disease Prevention and Control (ECDC). Migrant health: epidemiology of HIV and AIDS in migrant communities and ethnic minorities in EU/EEA countries [Internet]. Stockholm, Sweden; 2010. http://ecdc.europa.eu/en/publications/Publications/0907_ TER_Migrant_health_HIV_Epidemiology_review.pdf.

7. European Centre for Disease Prevention and Control WHO for Europe. HIV/AIDS surveillance in Europe 2014 [Internet]. Stockholm, Sweden; 2015. http://ecdc. europa.eu/en/publications/Publications/hiv-aids-surveillancein-Europe-2014.pdf.

8. Rice BD, Elford J, Yin Z, et al. A new method to assign country of HIV infection among heterosexuals born abroad and diagnosed with HIV. AIDS 2012; 26: 1961-1966.

9. Fakoya I, Álvarez-del Arco D, Woode-Owusu M, et al. A systematic review of post-migration acquisition of HIV among migrants from countries with generalised HIV epidemics living in Europe: implications for effectively managing HIV prevention programmes and policy. BMC Public Health 2015; 15: 561.

10. Hernando V, Alvárez-Del Arco D, Alejos B, et al. HIV infection in migrant populations in the European Union and European Economic Area in 2007-2012; an epidemic on the move. Journal of Acquired Immune Deficiency Syndromes 2015; 70: 204-211.

11. Deblonde J, Sasse A, Del Amo J, et al. Restricted access to antiretroviral treatment for undocumented migrants: a bottle neck to control the HIV epidemic in the EU/ EEA. BMC Public Health 2015; 15: 1228.

12. Ferrer L, Loureiro E, Meulbroek M, et al. High HIV incidence among men who have sex with men attending a community-based voluntary counselling and testing service in Barcelona, Spain: results from the ITACA cohort. Sexually Transmitted Infections 2015; 92: 70-75.

13. Tanser F, Bärnighausen $\mathbf{T}$, Vandormael A, et al. HIV treatment cascade in migrants and mobile populations. Current Opinion in HIV and AIDS 2015; 10: 430-438.

14. European Commission. Action Plan on HIV/AIDS in the EU and neighbouring countries: 2014-2016 [Internet]. 2014. http://ec.europa.eu/health/sti_prevention/docs/ec_ hiv_actionplan_2014_en.pdf.

15. European Commission. Commission decision of $28 / \mathrm{IV} /$ 2008 amending Decision 2002/253/EC laying down case definitions for reporting communicable diseases to the Community network under Decision No 2119/ 98/EC of the European Parliament and of the Council. [Internet]. Luxembourg; 2008 p. L1-79. http://ec.europa.eu/health/ph_threats/com/docs/1589_2008_en.pdf.

16. Antinori A, Coenen T, Costagiola D, et al. Late presentation of HIV infection: a consensus definition. HIV Medicine 2011; 12: 61-64.
17. Centre d'Estudis Epidemiològics sobre les Infeccions de Transmissió Sexual i Sida de Catalunya (CEEISCAT). Sistema integrat de vigilància epidemiològica de la SIDA/ VIH/ITS a Catalunya (SIVES) [Internet]. Barcelona, Spain; 2015. http://www.ceeiscat.cat/documents/sives2015.pdf.

18. World Health Organization. Consolidated strategic information guidelines for HIV in the health sector [Internet]. Geneva, Switzerland; 2015. http://www.who.int/hiv/pub/ guidelines/strategic-information-guidelines/en/.

19. Folch C, Casabona J, Espelt A, et al. Gender differences in HIV risk behaviours among intravenous drug users in Catalonia, Spain. Gaceta Sanitaria 2013; 27: 338-343.

20. Folch C, Casabona J, Sanclemente C, et al. [Trends in HIV prevalence and associated risk behaviors in female sex workers in Catalonia (Spain)]. Gaceta Sanitaria 2014; 28: 196-202.

21. Folch C, Fernández-Dávila $\mathbf{P}$, Ferrer L, et al. [High prevalence of drug consumption and sexual risk behaviors in men who have sex with men.]. Medicina Clinica 2015; 145: 102-107.

22. Fernàndez-Lopez $\mathbf{L}$, Rifà $\mathbf{B}$, Pujol $\mathbf{F}$, et al. Impact of the introduction of rapid HIV testing in the voluntary counselling and testing sites network of Catalonia, Spain. International Journal of STD \& AIDS 2010; 21: 388-391.

23. Jaén Á, Casabona J, Esteve A, et al. Clinical-epidemiological characteristics and antiretroviral treatment trends in a cohort of HIV infected patients. The PISCIS Project. Medicina Clinica 2005; 124: 525-531.

24. Generalitat de Catalunya; Institut d'Estadística de Catalunya. Estimacions de població. Dades postcensals. 2013. Barcelona; 2015.

25. Campbell CN, Ambrosioni J, Miro JM, et al. The continuum of HIV care in Catalonia. AIDS Care 2015; 27: 1449-1454.

26. Diez M, Bleda MJ, Varela JR, et al. Trends in HIV testing, prevalence among first-time testers, and incidence in most-at-risk populations in Spain: the EPI-VIH Study, 2000 to 2009. Eurosurveillance 2014; 19: 20971.

27. European Centre for Disease Prevention and Control (ECDC). Thematic report: migrants monitoring implementation of the Dublin declaration on partnership to fight HIV/AIDS in Europe and Central Asia: 2012 progress [Internet]. Stockholm, Sweden; 2013. http://ecdc. europa.eu/en/publications/Publications/dublin-declaration-monitoring-report-migrants-september-2013.pdf.

28. TAMPEP (European Network for HIVISTI Prevention and Health Promotion among Migrant Sex Workers). European overview of HIV and sex work [Internet]. Amsterdam; 2007. http://tampep.eu/documents/European OverviewofHIVandSexWork.pdf.

29. European Centre for Disease Prevention and Control (ECDC). Thematic report: sex workers; monitoring implementation of the Dublin declaration on partnership to fight HIV/AIDS in Europe and Central Asia: 2012 progress [Internet]. Stockholm, Sweden; 2013. http://ecdc.europa.eu/en/publications/Publications/dublin-declaration-sex-workers.pdf.

30. Diaz A, Junquera ML, Esteban V, et al. HIV/STI co-infection among men who have sex with men in Spain. Eurosurveillance 2009; 14: pii: 19426. 
31. Mimiaga MJ, Biello KB, Robertson AM, et al. High prevalence of multiple syndemic conditions associated with sexual risk behavior and HIV infection among a large sample of Spanish- and Portuguese-speaking men who have sex with men in Latin America. Archives of Sexual Behavior 2015; 44: 1869-1878.

32. Fernández-Dávila $\mathbf{P}$, Folch $\mathbf{C}$, Ferrer $\mathbf{L}$, et al. Who are the men who have sex with men in Spain that have never been tested for HIV? HIV Medicine 2013; 14 (Suppl 3): $44-48$.

33. Hoyos J, Fernández-Balbuena S, de la Fuente L, et al. Never tested for HIV in Latin-American migrants and Spaniards: prevalence and perceived barriers. Journal of the International AIDS Society 2013; 16: 18560.

34. Caro-Murillo AM, Castilla Catalán J, Del Amo Valero J. Epidemiología de la infección por VIH en inmigrantes en España: fuentes de información, características, magnitud y tendencias. Gaceta Sanitaria 2010; 24: 81-88.

35. Hernando Rovirola C, Ortiz-Barreda G, Galán Montemayor JC, et al. [HIV and other sexually transmitted infections among migrant population in Spain: a scoping review]. Revista Española de Salud Pública 2014; 88: 763-781.
36. Oliva J, Díez M, Galindo S, et al. Predictors of advanced disease and late presentation in new HIV diagnoses reported to the surveillance system in Spain. Gaceta Sanitaria 2014; 28: 116-122.

37. Van Beckhoven D, Florence E, Ruelle J, et al. Good continuum of HIV care in Belgium despite weaknesses in retention and linkage to care among migrants. $B M C$ Infectious Diseases 2015; 15: 496.

38. Royo-Bordonada MÁ, Díez-Cornell M, Llorente JM. Health-care access for migrants in Europe: the case of Spain. Lancet 2013; 382: 393-394.

39. Gil-González D, Carrasco-Portiño M, Vives-Cases C, et al. Is health a right for all? An umbrella review of the barriers to health care access faced by migrants. Ethnicity \& Health 2015; 20: 523-541.

40. Monge S, Alejos B, Dronda F, et al. Inequalities in HIV disease management and progression in migrants from Latin America and sub-Saharan Africa living in Spain. HIV Medicine 2013; 14: 273-283.

41. Thierfelder C, Weber R, Elzi L, et al. Participation, characteristics and retention rates of HIV-positive immigrants in the Swiss HIV Cohort Study. HIV Medicine 2012; 13: 118-126. 